\title{
Genetic and environmental contributions to serum ascorbic acid concentrations: the Stanislas Family Study
}

\author{
Bernard Herbeth $^{1,2 *}$, Jean-Claude Guilland ${ }^{3,4,5}$, Luc Rochette ${ }^{5}$, Gérard Siest ${ }^{1,2}$ \\ and Sophie Visvikis-Siest ${ }^{1,2}$ \\ ${ }^{1}$ INSERM U525, Nancy, F-54000 France \\ ${ }^{2}$ Université Henri Poincaré, Faculté de Pharmacie, Nancy, F-54000 France \\ ${ }^{3}$ Université de Bourgogne, Dijon, F-21000 France \\ ${ }^{4}$ Hôpital Général, Unité Neuromédiateurs et Vitamines, Dijon, F-21033 France \\ ${ }^{5}$ Laboratoire de Physiopathologie et Pharmacologie Cardiovasculaire Expérimentales, \\ Université de Bourgogne, Faculté de Médecine, Dijon, F-21079 France
}

(Received 11 April 2006 - Revised 5 September 2006 - Accepted 12 September 2006)

\begin{abstract}
Although numerous environmental factors are documented to influence serum ascorbic concentrations, little is known about the genetic versus environmental contributions to variation of this trait. The aim of this study was to estimate family correlation and, additive genetic heritability and household effects in a variance component analysis for serum ascorbic acid concentrations. In a sample of ninety French families, information was obtained regarding serum ascorbic acid concentrations, usual dietary intake, lifestyle, and other related covariates. Spouse, parent-offspring and offspring-offspring significant correlation coefficients for serum ascorbic acid concentrations, adjusted for age, cigarette consumption and oral contraceptive use, were 0.432, 0.298 and 0.485, respectively, and for adjusted values for additional diet covariates (vitamin C intake and fruit and vegetable consumption), were $0 \cdot 362,0 \cdot 154$ and $0 \cdot 348$, respectively. Variance component analysis for serum ascorbic concentrations showed no significant genetic contribution to variability of this trait. Conversely, household common environment accounted for 27.7 and $42.6 \%$ in parents and offspring, respectively, after adjustment for age, cigarette consumption and oral contraceptive use. After adjustment for the two additional diet covariates (vitamin $\mathrm{C}$ intake and fruit and vegetable consumption) household common variance decreased to 13.6 and $30.5 \%$ in parents and offspring, respectively. These results show that serum ascorbic acid concentrations aggregate within healthy families partly due to diet intake but without a significant genetic component.
\end{abstract}

Ascorbic acid: Serum concentration: Family resemblance: Genetics: Household environment

There is great interest in the clinical roles of vitamin $\mathrm{C}$ because of evidence that oxidative damage is a root cause of, or at least associated with, many degenerative diseases. Population studies show that individuals with high intakes of vitamin $\mathrm{C}$ and/or high blood levels have lower risk of a number of chronic diseases, including atherosclerosis and related cardiovascular complications, cancer, eye diseases and neurodegenerative conditions (Jacob \& Sotoudeh, 2002; Blomhoff, 2005). Data collected from nuclear families underlined the strong influence of family history on the risk of such various chronic diseases and the fact that these diseases are multifactorial in nature, being influenced both by genes, environmental factors and complex interactions (Risch, 2001; Grant, 2003; Flossmann et al. 2004; Lin et al. 2005). Other investigations showed that intermediate traits related to oxidative stress and antioxidant status themselves are multifactorial and influenced both by genetics and environment. There is strong evidence for genetic familial influences on total antioxidant activity (Wang et al. 2001), serum retinol and serum $\alpha$-tocopherol concentrations (Gueguen et al. 2005). However, data regarding the family components of circulating indices of vitamin $\mathrm{C}$ status are lacking.

The present study aimed to estimate family aggregation and, additive genetic heritability and household effects of serum ascorbic acid concentrations, accounting for the influence of known environmental covariates.

\section{Materials and methods}

Subjects and study design

This work is part of the Stanislas Family Study, a 10-year longitudinal follow-up study conducted since 1994 on 1006 families selected at the Centre for Preventive Medicine of Vandoeuvre-lès-Nancy (east of France) who were free of chronic or acute disease that could influence nutritional status and did not take vitamin supplementation (Siest et al. 1998). In this paper, we present data of the first examination

* Corresponding author: Dr Bernard Herbeth, INSERM U525, Faculté de Pharmacie, 30 rue Lionnois, 54000 Nancy, France, fax + 33 (0) 3 83 321322 , email Bernard.Herbeth@nancy.inserm.fr 
(1994-5) obtained from a random sub-sample of ninety families composed of two parents between 28 and 59 years and at least one child between 6 and 24 years (357 individuals). All subjects underwent a complete medical examination including weight and height measurements and blood samples, all the members of one family having this check-up on the same day. Alcohol consumption, smoking status and drug use were collected through validated questionnaires under the supervision of trained nurses. The research protocol was approved by the Comité Consultatif de Protection des Personnes dans la Recherche Biomédicale de Lorraine and each subject gave written informed consent.

\section{Dietary record}

Dietary intake was assessed by a $3 \mathrm{~d}$ dietary record (Vauthier et al. 1996), which was completed during two weekdays and one weekend day assigned at random for each family. All subjects received guidelines from a dietitian on the procedures to complete the dietary record and to measure food portions by using a weighing-scale. For young children, the $3 \mathrm{~d}$ diary was filled in by the mother and the child together. One week later, the $3 \mathrm{~d}$ record was checked and completed by the dietitian by using colour photographs of foods, each with three different portion sizes. Macronutrient and micronutrient intakes were estimated with an updated computerized version of the Répertoire général des aliments (Favier et al. 1995).

\section{Biological measurements}

A single venous blood sample was collected in a dry BD vacutainer tube (Vacutainer Tube; Becton Dickinson, Grenoble, France) from fasting individuals within $30 \mathrm{~min}$ for all members of one family. Blood samples were centrifuged at $2000 \mathrm{~g}$ for $15 \mathrm{~min}$ at $4^{\circ} \mathrm{C}$ and the resulting serum aliquots were stabilized by adding metaphosphoric acid $(5 \%$, w/v) immediately after separation from the cells $(1: 2, \mathrm{v} / \mathrm{v})$. The precipitates were removed by centrifugation $(2000 \mathrm{~g}$ for $15 \mathrm{~min}$ at $4^{\circ} \mathrm{C}$ ) and the samples were kept at $-80^{\circ} \mathrm{C}$ for $<6$ months until analysed. The stability of the metaphosphoric acid-treated serum samples has been thoroughly tested under these conditions (Comstock et al. 1995; Margolis \& Duewer, 1996). There were only slight losses during longterm freezer storage $(<1 \%$ per year).

Total vitamin $\mathrm{C}$ (ascorbic acid and dehydroascorbic acid) in metaphosphoric acid-treated serum was quantified using the method described previously (Tessier et al. 1996). Briefly, after enzymatic oxidation of ascorbic acid to dehydroascorbic acid, the latter was condensed with $o$-phenylenediamine to its quinoxaline derivative. This derivative was separated on a reversed-phase HPLC column and detected fluorometrically. The chromatographic system consisted of one Spectra Model P100 pump (Spectra Physics, Les Ulis, France), a Rheodyne 7125 injector and a $5 \mu \mathrm{m}$ Lichrospher $(15 \mathrm{~cm} \times 4.45 \mathrm{~cm}$ i.d. $)$ reversed-phase column (Merck, Darmstadt, Germany) protected by a $5 \mu \mathrm{m}$ reversed-phase guard column $(1 \mathrm{~cm} \times 0.45 \mathrm{~cm}$ i.d.; Merck). Detection of the quinoxaline derivative was achieved by using a Waters 2475 Multi $\lambda$ Fluorescence Detector (Waters, St Quentin-en-Yvelines, France). Excitation and emission wavelengths were set at 360 and $440 \mathrm{~nm}$, respectively. Peak areas were determined using a KromaSystem 2000 (BIO-TEK
Instruments, St Quentin-en-Yvelines, France). Mobile phase was $0.05 \mathrm{M}$-sodium phosphate containing $50 \%$ methanol, $\mathrm{pH}$ $7 \cdot 40$. The flow rate was set at $1.4 \mathrm{ml} / \mathrm{min}$.

The within-day and between-day precisions of the assay with respect to total vitamin $\mathrm{C}$ serum content were calculated from series of experiments made with aliquots of an average serum sample with a content of $38.2 \mu \mathrm{mol} / \mathrm{l}$. The within-day $\mathrm{CV}$ was $1.5 \%$ ( $n$ 10) while the between-day CV was calculated to be $2.5 \%$ ( $n$ 20, measured on separate days). These values are within acceptable limits. At a signal-to-noise ratio of 3 , the detection limit was calculated to be about $5 \mathrm{pmol} /$ $20 \mu \mathrm{l}$ injection. To ensure recovery from serum, aliquots from a single serum sample were spiked in duplicate with ascorbic acid to give an additional vitamin $\mathrm{C}$ concentration ranging from 5 to $100 \mu \mathrm{mol} / \mathrm{l}$. Recoveries across the whole range lay between 97.5 and $104.5 \%$. Internal quality assurance was monitored using Chromsystems vitamin $\mathrm{C}$ serum (Chromsystems Instruments \& Chemicals GmbH, Munich, Germany) and the laboratory participated in the external quality assurance programme from the Société Francophone Vitamines et Biofacteurs. Subjects were analysed in seven analytical runs (fifty to fifty-five subjects per run) and between-run precision of the assay was $2.5 \%$ during the present study.

\section{Statistical analysis}

Individual statistical analyses were performed by using the SAS software package version 9.1 (SAS Institute Inc., Cary, NC, USA). In the overall sample, stepwise multiple regression analysis was carried out to select significant covariates $(P \leq 0 \cdot 05)$ among the lifestyle factors and diet intakes listed in Table 1. Then regression coefficients were computed for the four sexby-generation groups (fathers, mothers, sons and daughters). In addition, significance of differences in regression coefficients between fathers, mothers, sons and daughters was assessed by testing interaction terms between each covariate and the four groups in the overall sample. As individuals within a family are not independent, statistical analyses were based on the estimating equation technique by using the SAS GENMOD procedure with a repeated statement. As the relationship between vitamin $\mathrm{C}$ intake and serum concentration has been hypothesized to be non-linear, the effect of vitamin $\mathrm{C}$ intake was tested in the regression analysis as continuous variables (both untransformed and $\log 10$-transformed values) and as a categorical variable in five classes $(0-49.9,50-99 \cdot 9,100-149.9,150-$ 199.9 and $>200 \mathrm{mg} / \mathrm{d})$.

Intra-familial correlations were estimated by using maximum likelihood techniques (Donner \& Koval, 1981) with and without adjustment for covariates. This statistical program allowed adjustment for covariates within models, simultaneously and separately for fathers, mothers, sons and daughters. The significance of various familial correlations or sex and generation differences in correlations was tested by using the log-likelihood ratio test. Correlations were computed under four sets of hypotheses: gender effects on correlations for parents and children (model 1), gender effects only for children (model 2), gender effects only for parents (model 3) and no gender effects at all (model 4).

Variance component analysis was applied in order to assess the relative contributions of genetic, common household factors and individual specific environment in family aggregation 
Table 1. Descriptive characteristics according to the four sex-by-generation groups $\dagger$ (Mean values and standard deviations)

\begin{tabular}{|c|c|c|c|c|c|c|c|c|}
\hline & \multicolumn{2}{|c|}{ Fathers $(n 90)$} & \multicolumn{2}{|c|}{ Mothers $(n 90)$} & \multicolumn{2}{|c|}{ Sons $(n 78)$} & \multicolumn{2}{|c|}{ Daughters ( $n$ 99) } \\
\hline & Mean & SD & Mean & SD & Mean & SD & Mean & SD \\
\hline Age (years) & $42 \cdot 2$ & $5 \cdot 2$ & 40.5 & 4.9 & $14 \cdot 1$ & 3.4 & $14 \cdot 3$ & 4.3 \\
\hline $\mathrm{BMI}\left(\mathrm{kg} / \mathrm{m}^{2}\right)$ & $25 \cdot 7$ & $3 \cdot 1$ & $23 \cdot 3$ & $3 \cdot 4$ & $19 \cdot 3$ & 3.0 & $19 \cdot 7$ & 3.3 \\
\hline Alcohol consumption $(\mathrm{g} / \mathrm{d})$ & 21.8 & $26 \cdot 1$ & 4.9 & 8.2 & 1.3 & 5.4 & 0.6 & $2 \cdot 1$ \\
\hline Tobacco consumption (cigarettes/d) & 5.4 & 10.5 & 3.1 & 6.9 & 0.6 & 2.9 & 0.8 & 3.2 \\
\hline Oral contraceptive use (\%) & - & - & $21 \cdot 1$ & - & - & - & $17 \cdot 1$ & - \\
\hline Serum ascorbic acid ( $\mu \mathrm{mol} / \mathrm{l})$ & 31.8 & $15 \cdot 4$ & $38 \cdot 8$ & $16 \cdot 7$ & 39.9 & $18 \cdot 3$ & $45 \cdot 6$ & $18 \cdot 8$ \\
\hline \multicolumn{9}{|l|}{ Diet intake } \\
\hline Energy $(\mathrm{kJ})$ & 10070 & 2160 & 7540 & 1730 & 10240 & 2450 & 8250 & 1800 \\
\hline Protein (\%)‡ & 18.5 & 2.9 & 18.9 & $4 \cdot 1$ & $16 \cdot 2$ & $2 \cdot 2$ & $16 \cdot 3$ & 3.0 \\
\hline Carbohydrate $(\%) \ddagger$ & 44.7 & $6 \cdot 5$ & 44.4 & $7 \cdot 3$ & $48 \cdot 4$ & $5 \cdot 6$ & $46 \cdot 7$ & $5 \cdot 6$ \\
\hline Fat $(\%) \ddagger$ & $36 \cdot 8$ & $5 \cdot 5$ & $36 \cdot 7$ & $6 \cdot 0$ & $35 \cdot 3$ & $5 \cdot 3$ & 36.9 & 5.4 \\
\hline Fruit and vegetable $(\mathrm{g} / \mathrm{d})$ & 328 & 188 & 319 & 183 & 223 & 135 & 238 & 128 \\
\hline Vitamin C $(\mathrm{mg} / \mathrm{d})$ & $79 \cdot 7$ & 55.9 & $75 \cdot 8$ & $46 \cdot 7$ & $76 \cdot 1$ & 39.7 & 88.9 & 52.7 \\
\hline Vitamin C $(\mathrm{mg} / 1000 \mathrm{~kJ}) \S$ & 8.4 & $6 \cdot 7$ & $10 \cdot 4$ & $6 \cdot 8$ & $7 \cdot 7$ & $4 \cdot 2$ & $11 \cdot 1$ & $6 \cdot 6$ \\
\hline
\end{tabular}

†For details of procedures, see p. 1014.

$\ddagger$ Expressed as percentage of non-alcohol energy intakes.

$\S$ Nutritional densities: vitamin intakes $(\mathrm{mg} / \mathrm{d}) /$ energy intakes $(\mathrm{kJ} / \mathrm{d})$.

of serum ascorbic acid concentrations. We used crude values and adjusted variables for significant covariates, separately for fathers, mothers, sons and daughters. The analysis was conducted by using a multivariate normal model for pedigree analysis as described by Lange et al. $(1976,1988)$ with the software FISHER which also performed tests of goodnessof-fit of the underlying multinormal distribution. The general model assumed that the studied trait was the result of the sum of three independent random components: a polygenic component $(\mathrm{G})$ representing additive genetic factors, household factors common to individuals within a family $(\mathrm{H})$ and unmeasured environmental factors particular to an individual (including measurement error) (E). These three components were assumed to be normally distributed with mean equal to 0 and variance equal to $\sigma_{\mathrm{G}}^{2}, \sigma_{\mathrm{H}}^{2}$ and $\sigma_{\mathrm{E}}^{2}$, respectively.

The hypothesis of no polygenic component or no household effect was checked by comparing a model including $\sigma_{\mathrm{G}}^{2}, \sigma_{\mathrm{H}}^{2}$ and $\sigma_{\mathrm{E}}^{2}$ with a model including only $\sigma_{\mathrm{H}}^{2}$ and $\sigma_{\mathrm{E}}^{2}$ or $\sigma_{\mathrm{G}}^{2}$ and $\sigma_{\mathrm{E}}^{2}$, respectively. Comparison of nested models was based on the likelihood ratio criteria. Eventually, the best parsimonious model was selected. The contribution (as a percentage) of the three components, additive genetic factors (heritability), household factors and residual environmental, to residual phenotypic variance (after adjustment for covariates) was deduced.

\section{Results}

Descriptive characteristics, serum ascorbic acid concentrations, and daily intakes of energy, macronutrients, fruits and vegetables, and vitamin $\mathrm{C}$ for the four sex-by-generation groups are summarized in Table 1.

Predictors of serum ascorbic acid concentrations in parents and offspring are presented in Table 2 . The proportion of phenotypic variance accounted for by the measured covariates was $29.1 \%$ in the overall group and ranged from $18.6 \%$ (in fathers) to $35.4 \%$ (in mothers). In the overall sample, females had significantly higher concentrations of ascorbic acid than males, and vitamin $\mathrm{C}$ intake and fruit and vegetable consumption were significantly and positively related to serum concentrations. Conversely, age, oral contraceptive use and smoking were negatively related to serum levels. Separating the four sex-by-generation groups, age in the four groups, smoking in fathers and oral contraceptive use in mothers were negative significant predictors of serum ascorbic acid concentrations. Vitamin $\mathrm{C}$ intakes in the four groups and fruit and vegetable consumption in mothers and sons were positively and significantly related to ascorbate concentration. Vitamin $\mathrm{C}$ intake was tested in regression analysis as continuous variables (both untransformed and log10-transformed values) and as a categorical variable in five classes. Among the three tested variables, untransformed vitamin $\mathrm{C}$ intake contributed better to the model after adjustment for covariates ( $F$ values were 32.84, 32.08 and 9.98 for untransformed, $\log 10$-transformed and categorical variables, respectively). In addition, interactions between each covariate and the four subgroups were not statistically significant.

The patterns of family correlations for serum ascorbic acid concentrations are given in Table 3 for crude and adjusted values. By using crude values, significant correlations $(P \leq 0.01$ and $P \leq 0.001)$ were found for all the various pairs of relatives and the hypothesis that no familial resemblance exists within the families was rejected $(P \leq 0.001$, data not shown). The hypothesis of no difference in correlations for father-offspring and mother-offspring (FS $=\mathrm{MS}$ and $\mathrm{FD}=\mathrm{MD}$ ), but with different correlations according to child gender, was tested with model 2. Model 3 assumed no effect of child gender on family correlations $(\mathrm{FS}=\mathrm{FD}, \mathrm{MS}=\mathrm{MD}$ and $\mathrm{SS}=\mathrm{SD}=\mathrm{DD})$. Model 4 hypothesized no effect of gender on family correlations ( $\mathrm{FS}=\mathrm{MS}=\mathrm{MD}=\mathrm{FD}$ and $\mathrm{SS}=\mathrm{SD}=\mathrm{DD})$. The most adequate parsimonious model was model 4. Spouse, parent-offspring and offspring-offspring correlation coefficients were $0.432,0.349$ and 0.514 , respectively. After adjustment for age, smoking and oral contraceptive use, correlation coefficients decreased for parentoffspring and offspring-offspring pairs to 0.298 and 0.485 , respectively. Additional adjustment for diet intakes (vitamin C, fruits and vegetables) led to lower correlation coefficients, $0.362,0.154$ and 0.348 , for the three pairs of 
relatives, respectively. Model 6 that took into account the six covariates was the most parsimonious $(P \leq 0 \cdot 001)$.

Quantitative genetic analyses were performed for serum ascorbic acid concentrations on crude values and after adjustment for the covariates previously described in Table 2 . Table 4 shows the components of variance attributable to additive genetic effects, shared household effects and residual environmental factors (including assay imprecision). By using crude values, the full model 1 including nine components $\left(\sigma_{\mathrm{G}}^{2}\right.$, and $\sigma_{\mathrm{H}}^{2}$ and $\sigma_{\mathrm{E}}^{2}$, specific of fathers, mothers, sons and daughters) was rejected by log-likelihood tests. No significant genetic contribution to variability in ascorbate concentration was found. The model giving the best description of the variance decomposition included two components of household effects for spouses and offspring, respectively, and only one component attributable to residual environment factors independently of gender and generation (model 4). The proportion of phenotypic variability accounted for by household was smaller in spouses than in children $(30.7 \%$ v. $47.5 \%)$. When values were adjusted for age, smoking and oral contraceptive use, spouse and child household common variances were 27.7 and $42.6 \%$, respectively. Model 7 , including adjustment for age, smoking, oral contraceptive use, and vitamin $\mathrm{C}$ and fruit and vegetable intakes, was considered to give the best description of the variance decomposition: spouse and child household common variances decreased to 13.6 and $30.5 \%$, respectively.

\section{Discussion}

Up to the present time no data have been available regarding the family aggregation of serum concentrations of ascorbic acid. Since the present study was done on a random subsample of ninety families of the Stanislas population, conclusions drawn from this subsample should be valid only for families living in the east of France with similar characteristics. Comparisons of the present results with those of studies to come should take into account the characteristics of these French nuclear families.

In the present study, spouse, parent-offspring and sibling correlation coefficients were significant with no difference between fathers and mothers or between sons and daughters. Effects of age and lifestyle-related covariates previously reported in the literature - smoking, oral contraceptive use and diet (Horwitt et al. 1975; Rivers, 1975; Webb, 1980; Hercberg et al. 1994; Marangon et al. 1998; Alberg, 2002; Galan et al. 2005) - substantially modified these family correlations. In particular, after adjustment for diet, both vitamin C intakes and fruit and vegetable consumption, regression coefficients decreased by 0.070 for spouse pairs $(0.432$ to 0.362$)$ and by about 0.14 for parent-offspring and offspring-offspring pairs $(0.298$ to 0.154 and 0.485 to 0.348 , respectively). In the same way, variance component analysis documents the importance of household effect (cohabitation and cultural inheritance) that was higher in children than in spouses, $47.5 \%$ v. $30.7 \%$, respectively. When models were adjusted for lifestyle covariates, household effect estimates were lower than in models without adjustment, $13.6 \% \mathrm{v}$. $30.7 \%$ and $30.5 \%$ v. $47.5 \%$, for spouses and children, respectively. Particularly, after adjustment for diet, household variance decreased by $12.0 \%$ to $14.1 \%$. In addition, adjustment for covariates did not substantially change residual variance. In adults and children in the present study, correlations between dietary 
Table 3. Estimates of familial correlations for serum ascorbic acid concentrations $\dagger$

\begin{tabular}{|c|c|c|c|c|c|c|}
\hline & Model 1 & Model 2 & Model 3 & Model 4 & Model 5 & Model 6 \\
\hline FM & $0.432^{\star \star \star}$ & $0.432^{\star \star \star}$ & $0.432^{\star \star \star}$ & $0.432^{\star \star \star}$ & $0.432^{\star \star \star}$ & $0.362^{\star \star \star}$ \\
\hline FS & $0.332^{\star \star *}$ & $0.388^{\star \star \star}$ & $0.319^{\star \star \star}$ & $0.349^{\star \star \star}$ & $0.298^{\star \star \star}$ & $0.154^{\star}$ \\
\hline FD & $0.291^{\star *}$ & $0.313^{\text {***}}$ & {$[0.319]$} & [0.349] & [0.298] & {$[0.154]$} \\
\hline MS & $0.447^{\star \star *}$ & {$[0.313]$} & $0.379^{\star \star *}$ & {$[0.349]$} & {$[0.298]$} & {$[0.154]$} \\
\hline $\mathrm{MD}$ & $0.334^{* * *}$ & {$[0.313]$} & {$[0.379]$} & [0.349] & {$[0.298]$} & {$[0.154]$} \\
\hline SS & $0.602^{* * *}$ & $0.595^{\star \star *}$ & $0.515^{\star * *}$ & $0.514^{\star \star *}$ & $0.485^{\star \star \star *}$ & $0.348^{\star \star \star}$ \\
\hline SD & $0.455^{\star * *}$ & $0.465^{\star * * *}$ & {$[0.515]$} & {$[0.514]$} & {$[0.485]$} & {$[0.348]$} \\
\hline DD & $0.535^{\star \star *}$ & $0.531^{* * *}$ & {$[0.515]$} & {$[0.514]$} & {$[0.485]$} & {$[0.348]$} \\
\hline$-\log _{e} L \neq$ & $1490 \cdot 23$ & 1490.67 & 1491.04 & 1491.27 & 1477.39 & 1449.28 \\
\hline Alternate model & - & Model 1 & Model 1 & Model 1 & Model 4 & Model 4 \\
\hline$x^{2}, d f \S$ & _- & $0.88,2$ & $1.62,4$ & $2 \cdot 08,5$ & $27 \cdot 76,16$ & $83.98,24$ \\
\hline$\hat{P S}$ & - & NS & NS & NS & $\leqslant 0.05$ & $\leqslant 0.001$ \\
\hline
\end{tabular}

FM, father-mother; FS, father-son; FD, father-daughter; MS, mother-son; MD, mother-daughter, SS, son-son; SD, son-daughter; DD, daughter-daughter.

Values were significantly different compared to zero: ${ }^{*} P \leq 0.05 ;{ }^{* *} P \leq 0.01 ;{ }^{* *} P \leq 0.001$.

† Models 1 to 4 used crude values: model 1 estimated all eight correlations, model 2 (no gender effect on parents correlations: FS $=$ MS and $F D=M D$ ), model 3 (no gender effect on offspring correlations: $F S=F D, M S=M D$ and $S S=S D=D D$ ), model 4 (no gender effect on parents nor offspring correlations: $F S=M S=M D=F D$ and $S S=S D=D D$ ). Model 5 used adjusted values for age, cigarette consumption and oral contraceptive use and estimated three correlation coefficients as model 4 (no gender effect on parents nor offspring correlations: $\mathrm{FS}=\mathrm{MS}=\mathrm{MD}=\mathrm{FD}$ and $\mathrm{SS}=\mathrm{SD}=\mathrm{DD}$ ). Model 6 used adjusted values for age, cigarette consumption, oral contraceptive use, vitamin $C$ intake, and fruit and vegetable consumption and estimated three correlation coefficients as model 4 (no gender effect on parents nor offspring correlations: $F S=M S=M D=F D$ and $S S=S D=D D$ ). Values in square brackets were constrained to be equal to a preceding value according to the hypotheses of the sub-model.

$\mp$ Logarithm of likelihood function.

$\S$ Likelihood ratio test.

and serum ascorbic acid concentrations were high and consistent with those found in the literature (Hercberg et al. 1994; Marangon et al. 1998; Galan et al. 2005) and explain partly the family aggregation. However, residual household variances in both generations remained after adjustment (13.6\% and $30.7 \%$ ) and may result from shared household behaviour not taken into account in the present analysis.

Contrary to fat-soluble vitamins (Gueguen et al. 2005) or plasma total antioxidant activity, an indicator of redox homeostasis (Wang et al. 2001), the heritability (additive genetic factors) estimate for serum ascorbic acid concentrations was low and non-significant. In the current study, genetics of proteins and enzymes that are involved in ascorbate metabolism did not seem to be implicated in family aggregation. Vitamin $\mathrm{C}$ concentrations in plasma and tissues are tightly controlled in man as a function of dose. Tight control is mediated by intestinal absorption, tissue distribution and renal reabsorption. Vitamin $\mathrm{C}$ is transported and accumulated in two ways. In one mechanism, ascorbate is directly transported across membranes by two sodium-dependent vitamin $\mathrm{C}$ transporter proteins: SVCT1 (encoded by SLC23A1 and mapped to 5q31.2) and SVCT2 (encoded by SLC23A2 and mapped to 20p13) (Stratakis et al. 2000). In the second mechanism, dehydroascorbic acid, which is oxidized ascorbic acid, is transported and reduced upon entry (Wang et al. 1997). This mechanism is sodium-independent and is mediated by GLUT transporters (Vera et al. 1993). The dominant mechanism of vitamin C accumulation is sodium-dependent transport by SVCT1 (SLC23A1) and SVCT2 (SLC23A2), which control vitamin C absorption, tissue accumulation and renal reabsorption. SLC23A1 expression has been located to the intestine, liver and kidney, sites of absorption and reabsorption across the epithelium. SLC23A2 expression occurs in adrenal, bone, brain, haematopoietic, lung and pituitary cell types, and probably mediates most vitamin $\mathrm{C}$ accumulation (Tsukaguchi et al. 1999). Comparison of genomic structure and variation in the two human sodium-dependent vitamin $\mathrm{C}$ transporters show single nucleotide polymorphisms in SLC23A1, which are population-specific in either African Americans or Caucasians, including three or four non-synonymous single nucleotide polymorphisms (Eck et al. 2004). Most single nucleotide polymorphisms in SLC23A2 are shared between African Americans and Caucasians, and there are no non-synonymous single nucleotide polymorphisms in SLC23A2 (Eck et al. 2004). If dose-concentration curves are shifted toward increased excretion and/or decreased absorption because of genomic variation in SLCA23A1, there could be substantial impact on vitamin $\mathrm{C}$ concentration in plasma. Lack of significant genetic influence in family aggregation within the Stanislas cohort suggests that genetic variation in SLC23A1 or $S L C 23 A 2$ has a weak effect on vitamin $\mathrm{C}$ metabolism in healthy subjects.

However, there could be some criticism about the failure to find significant heritable influences on serum ascorbic acid concentrations. By using family design, estimates of effects attributable to genes and to shared households depend on numerous factors such as types of related individuals, accuracy of a single measurement of serum ascorbic acid (particularly, the adequacy to characterize long-term vitamin status), inclusion of subjects with extreme values of variables, distribution of environment factors (for instance the range of vitamin intakes from foods and/or supplements), covariates were included in the model and type of expression of variance components (proportion of the total variance or of residual variance).

According to a previous experiment, the number of samples required to characterize accurately long-term plasma levels of ascorbic acid of an individual ranged from 1.1 to 4.4 (95\% CI of 2.4 samples; Cooney et al. 1995). In the present study, due to design restrictions, only one single blood sample was obtained per subject. However, in order to reduce intra-subject variability of this biological trait and consequently to improve 
Table 4. Variance components of serum ascorbic acid concentrations $\dagger$

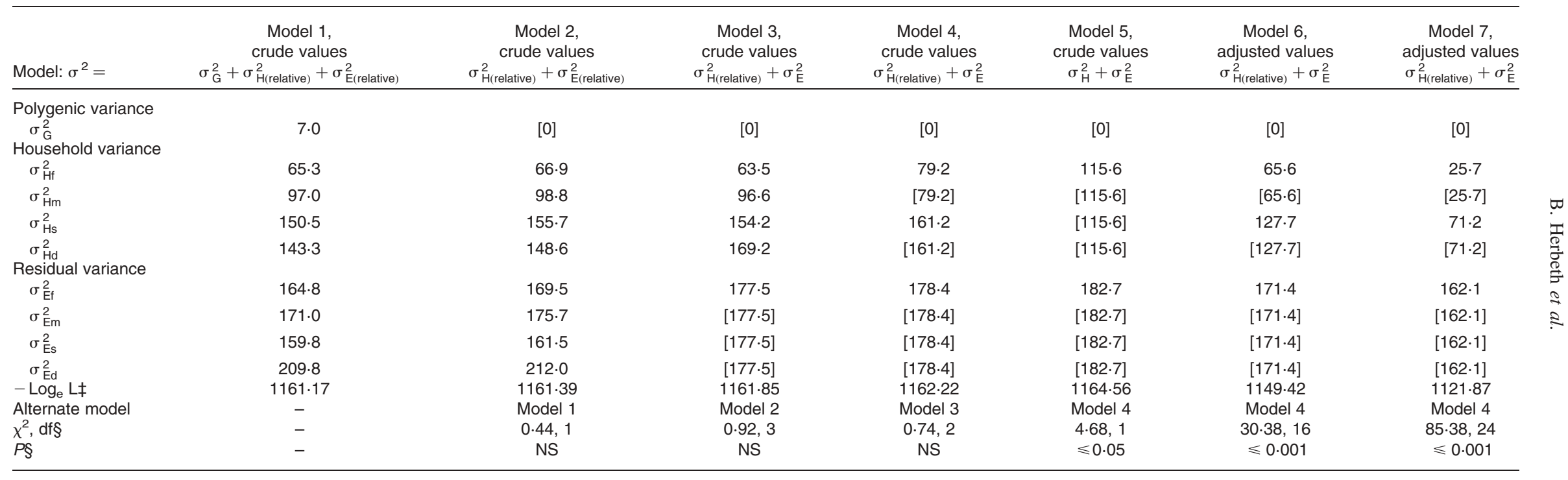

$\sigma_{\mathrm{G}}^{2}$, additive polygenic variances; $\sigma_{\mathrm{Hf}}^{2}, \sigma_{\mathrm{Hm}}^{2}, \sigma_{\mathrm{Hs}}^{2}, \sigma_{\mathrm{Hd}}^{2}$, father, mother, son, daughter household common variances; $\sigma_{\mathrm{Ef}}^{2}, \sigma_{\mathrm{Em}}^{2}, \sigma_{\mathrm{Es}}^{2}, \sigma_{\mathrm{Ed}}^{2}$, father, mother, son, daughter residual variances.

t Models 1 to 5 used crude values. Model 6 used adjusted values for age, cigarette consumption and oral contraceptive use. Model 7 used adjusted values for age, cigarette consumption, oral contraceptive use, vitamin $\mathrm{C}$ intake, and fruit and vegetable consumption.

¥Logarithm of likelihood function. Values in square brackets were fixed or constrained to be equal to a preceding value according to model hypotheses. $\S$ Likelihood ratio test. 
the statistical power of component analysis, inter-assay variability was minimized ( $2.5 \%$ in the present study), temporal variations (especially seasonal) within family were lacking (blood samples were collected within 30 min for all members of one family), and adjusted values for factors that influence circulating levels such as dietary intake and oral contraceptive use were used.

In addition, variance component analysis requires strict assumptions such as additive effects of multiple genes, environment factors, family environment and cultural transmission (Susser \& Susser, 1987). The assumptions of these models may have a marked effect on their results, particularly the tendency to overestimate heritability or environmental effect because of three main issues: (1) genotypic variance may include shared lifestyle variance that has not been removed by design or analysis and, vice versa, (2) estimates could greatly differ across populations according to the distribution of environmental and genetic factors, and (3) the assumption of independence between genotype and environment is likely to be violated when covariation and interaction are present.

However, taking into account such limitations, the general finding of the present study was that family aggregation in serum ascorbic acid concentrations was significant within Stanislas families. Slight and not significant genetic influence was shown in family aggregation and household effect was higher in children than in spouses. Furthermore, adjustment for lifestyle covariates such as smoking habits, oral contraceptive and diet intake, both vitamin $\mathrm{C}$ and, fruit and vegetables, contributed to a decreased cohabitation and cultural inheritance effect in offspring and spouses. Such family aggregation could partly explain the strong influence of family history on the risk of various chronic diseases such atherosclerosis and related cardiovascular complications, cancer, eye diseases and neurodegenerative conditions. Since familial habits act on family resemblance in diet-related blood indices, family should be a favourite unit for health and diet promotion programmes.

\section{Acknowledgements}

None of the authors had any financial or personal conflict of interest. B. H. designed this specific study, performed the statistical analysis, interpreted the data and wrote the paper. J.-C. G. was responsible for laboratory analyses and participated in writing the paper. G. S. and S. V. are the principal investigators of the Stanislas Family Study. We are deeply grateful for the co-operation of the families participating in the Stanislas Cohort. We acknowledge the management, reception, preclinical, laboratory and medical staff of the Centre for Preventive Medicine of Vandoeuvre-lès-Nancy (France). We especially thank Sylvie Péchiné for collection of food intake data, Maryvonne Chaussard, Chantal Lafaurie and Véronique Michaud (Nancy) for family recruitment, Françoise Duchenne and Marie-Claude Riard (Dijon) for technical assistance with the biochemical assays, and Edith Lecomte who co-ordinated the field work. We also thank David Trégouët (INSERM U 525, Paris, France) and René Gueguen (Centre de Médicine Préventive, Vandoeuvre-lès-Nancy, France) for providing the genetic analysis software. Frozen aliquots of serum were stored in the bio-bank of the Centre de Médecine Préventive (Vandoeuvre-lès-Nancy, France).

\section{References}

Alberg A (2002) The influence of cigarette smoking on circulating concentrations of antioxidant micronutrients. Toxicology $\mathbf{1 8 0}$, $121-137$

Blomhoff R (2005) Dietary antioxidants and cardiovascular disease. Curr Opin Lipidol 16, 47-54.

Comstock GW, Norkus EP, Hoffman SC, Xu MW \& Helzlsouer KJ (1995) Stability of ascorbic acid, carotenoids, retinol, and tocopherols in plasma stored at -70 degrees $\mathrm{C}$ for 4 years. Cancer Epidemiol Biomarkers Prev 4, 505-507.

Cooney RV, Franke AA, Hankin JH, Custer LJ, Wilkens LR, Harwood PJ \& Le Marchand L (1995) Seasonal variations in plasma micronutrients and antioxidants. Cancer Epidemiol Biomarkers Prev 4, 207-215.

Donner A \& Koval JJ (1981) A multivariate analysis of family data. Am J Epidemiol 114, 149-154.

Eck P, Erichsen HC, Taylor JG, Yeager M, Hughes AL, Levine M \& Chanock S (2004) Comparison of the genomic structure and variation in the two human sodium-dependent vitamin $\mathrm{C}$ transporters, SLC23A1 and SLC23A2. Hum Genet 115, 285-294.

Favier G, Ireland-Ripert J, Toque C \& Feinberg M (1995) Répertoire général des aliments. Composition Tables, 2nd ed., Paris: INRA Technique et Documentation.

Flossmann E, Schulz UG \& Rothwell PM (2004) Systematic review of methods and results of studies of the genetic epidemiology of ischemic stroke. Stroke 35, 212-227.

Galan P, Viteri FE, Bertrais S, et al. (2005) Serum concentrations of beta-carotene, vitamins $\mathrm{C}$ and $\mathrm{E}$, zinc and selenium are influenced by sex, age, diet, smoking status, alcohol consumption and corpulence in a general French adult population. Eur J Clin Nutr 59, $1181-1190$.

Grant PJ (2003) The genetics of atherothrombotic disorders: a clinician's view. J Thromb Haemost 1, 1381-1390.

Gueguen S, Leroy P, Gueguen R, Siest G, Visvikis S \& Herbeth B (2005) Genetic and environmental contributions to serum retinol and alpha-tocopherol concentrations: the Stanislas Family Study. Am J Clin Nutr 81, 1034-1044.

Hercberg S, Preziosi P, Galan P, Devanlay M, Keller H, Bourgeois C, Potier DC \& Cherouvrier F (1994) Vitamin status of a healthy French population: dietary intakes and biochemical markers. Int $J$ Vitam Nutr Res 64, 220-232.

Horwitt MK, Harvey CC \& Dahm CH Jr (1975) Relationship between levels of blood lipids, vitamins $\mathrm{C}$, A, and E, serum copper compounds, and urinary excretions of tryptophan metabolites in women taking oral contraceptive therapy. Am J Clin Nutr 28, 403-412.

Jacob RA \& Sotoudeh G (2002) Vitamin C function and status in chronic disease. Nutr Clin Care 5, 66-74.

Lange K, Weeks D \& Boehnke M (1988) Programs for pedigree analysis: MENDEL, FISHER, and dGENE. Genet Epidemiol 5, $471-472$.

Lange K, Westlake J \& Spence MA (1976) Extensions to pedigree analysis. III. Variance components by the scoring method. Ann Hum Genet 39, 485-491.

Lin HF, Boden-Albala B, Juo SH, Park N, Rundek T \& Sacco RL (2005) Heritabilities of the metabolic syndrome and its components in the Northern Manhattan Family Study. Diabetologia 48, 2006-2012.

Marangon K, Herbeth B, Lecomte E, Paul-Dauphin A, Grolier P, Chancerelle Y, Artur Y \& Siest G (1998) Diet, antioxidant status, and smoking habits in French men. Am J Clin Nutr 67, 231-239.

Margolis SA \& Duewer DL (1996) Measurement of ascorbic acid in human plasma and serum: stability, intralaboratory repeatability, and interlaboratory reproducibility. Clin Chem 42, 1257-1262.

Risch N (2001) The genetic epidemiology of cancer: interpreting family and twin studies and their implications for molecular 
genetic approaches. Cancer Epidemiol Biomarkers Prev 10, $733-741$.

Rivers JM (1975) Oral contraceptives and ascorbic acid. Am J Clin Nutr 28, 550-554.

Siest G, Visvikis S, Herbeth B, et al. (1998) Objectives, design and recruitment of a familial and longitudinal cohort for studying gene-environment interactions in the field of cardiovascular risk: the Stanislas cohort. Clin Chem Lab Med 36, 35-42.

Stratakis CA, Taymans SE, Daruwala R, Song J \& Levine M (2000) Mapping of the human genes (SLC23A2 and SLC23A1) coding for vitamin C transporters 1 and 2 (SVCT1 and SVCT2) to $5 \mathrm{q} 23$ and 20p12, respectively. J Med Genet 37, E20.

Susser M \& Susser E (1987) Indicators and designs in genetic epidemiology: separating heredity and environment. Rev Epidemiol Sante Publique 35, 54-77.

Tessier F, Birlouez-Aragon I, Tjani C \& Guilland JC (1996) Validation of a micromethod for determining oxidized and reduced vitamin $\mathrm{C}$ in plasma by HPLC-fluorescence. Int $J$ Vitam Nutr Res 66, 166-170.
Tsukaguchi H, Tokui T, Mackenzie B, Berger UV, Chen XZ, Wang Y, Brubaker RF \& Hediger MA (1999) A family of mammalian $\mathrm{Na}^{+}$-dependent L-ascorbic acid transporters. Nature 399, $70-75$.

Vauthier JM, Lluch A, Lecomte E, Artur Y \& Herbeth B (1996) Family resemblance in energy and macronutrient intakes: the Stanislas Family Study. Int $J$ Epidemiol 25, $1030-1037$.

Vera JC, Rivas CI, Fischbarg J \& Golde DW (1993) Mammalian facilitative hexose transporters mediate the transport of dehydroascorbic acid. Nature 364, 79-82.

Wang XL, Rainwater DL, VandeBerg JF, Mitchell BD \& Mahaney MC (2001) Genetic contributions to plasma total antioxidant activity. Arterioscler Thromb Vasc Biol 21, 1190-1195.

Wang Y, Russo TA, Kwon O, Chanock S, Rumsey SC \& Levine M (1997) Ascorbate recycling in human neutrophils: induction by bacteria. Proc Natl Acad Sci U S A 94, 13816-13819.

Webb JL (1980) Nutritional effects of oral contraceptive use: a review. J Reprod Med 25, 150-156. 